\title{
Blended learning y realidad aumentada: experiencias de diseño docente
}

\section{Blended learning and augmented reality: experiences of educational design}

\author{
Julio Cabero Almenara \\ Universidad de Sevilla (España) \\ Verónica Marín-Díaz \\ Universidad de Córdoba (España)
}

\section{Resumen}

La formación a distancia pasa en estos momentos por una redefinición de conceptos, entre otros motivos por el auge que las denominadas tecnologías emergentes, están adquiriendo, ampliando, de esta forma, las tecnologías con las cuales los alumnos pueden interaccionar $\mathrm{y}$ las experiencias que se pueden ofrecer. El tradicional y actual formato blended learning que domina la enseñanza universitaria formada por lo general, por una combinación de no presencialidad, flexibilidad y facilidad de acceso, está demandando una nueva visión y forma de empleo. Junto a ella la Realidad Aumentada, catalogada por el Informe Horizon como una tecnología que debe estar presente en los sistemas educativos en torno al año 2020, se presenta como un recurso que puede potenciar al anterior, dada la cercanía entre contenido y realidad de contexto que presenta, gracias a su posibilidad de ser empleado a distancia. En este artículo se hace un análisis de ambos conceptos, así como se presenta la posibilidad de imbricación del segundo con el primero. Para constatar un ejemplo de la combinación de ambos, se expone el trabajo realizado desde el proyecto RAFODIUM, en los Grados de Pedagogía y Medicina de la Universidad de Sevilla, mediante la descripción de los objetos creados a través de Realidad Aumentada y apuntes enriquecidos que pueden ser consultados en la plataforma de teleformación que la citada universidad emplea. Se concluye este artículo señalando que la formación on-line reclama acciones formativas atractivas y motivadoras conectadas con la realidad, aspecto que la Realidad Aumentada aporta.

Palabras clave: educación a distancia; blended learning; estilos de aprendizaje; realidad aumentada; diseño de materiales.

\section{Abstract}

Nowadays, Distance learning goes through the redefinition of concepts, among other reasons by the rise that the so-called emerging technologies are acquiring, thus expanding the technologies with which the students can interact and the experiences that can be provided. The traditional and current format blended learning which dominates the university education 
generally formed by a combination of non-presence, flexibility and easy access, is demanding a new view and form of usage. Together with it, the Augmented Reality, catalogued by the Horizon Report as a technology which must be present in the educational system about the year 2020, is presented as a resource which can improve the previous one, given the closeness between the content and the context reality presented, thanks to its possibility of being used at a distance. In this article an analysis of both concepts is carried out, as well as the possibility of overlapping the second within the first one is presented. In order to verify an example of the combination of both, we present the work carried out from the RAFODIUM project, in the Pedagogy and Medical Degrees at the University of Seville, by means of the description of objects created by Augmented Reality and of the enriched notes which can be consulted through the learning management platform that the mentioned university uses. The article is concluded by highlighting that the on-line training demands attractive and motivating formative actions connected with the reality, an aspect that the Augmented Reality offers.

Keywords: distance study; blended learning; learning styles; augmented reality; material design.

Hablar de blended learning y realidad aumentada (RA), es referirnos a dos propuestas tecnológicas que están presentando un gran impacto en los últimos tiempos en distintos sectores educativos, como han puesto de manifiesto diferentes Informes Horizon (Johnson, Adams, Gago, Garcia, y Martín, 2013; Johnson, Adams, Cummins, Estrada, Freeman y Hall, 2016); más concretamente en el último de ellos se indica:

A partir de las mejores prácticas en línea método cara a cara, el blended learning está en aumento en las universidades y colegios. Las ventajas de las ofertas de aprendizaje combinado son ahora bien conocidas, y su flexibilidad, facilidad de acceso y la integración de sofisticados medios y tecnologías son evidentes (NMC, 2017, p. 4).

Idéntica situación de plantearlas como tecnologías de alta penetración en la formación universitaria la encontramos en informes realizados por el Observatorio de Innovación Educativa del Instituto Tecnológico de Monterrey (Tecnológico de Monterrey, 2015).

Lo expuesto, nos lleva a señalar que son dos propuestas que están comenzando a consolidarse en el sistema educativo, aunque, lógicamente, por su antigüedad, las acciones de b-learning, se encuentran más asentadas, tanto conceptual como empíricamente (Llorente y Cabero, 2009; Marín, Reche y Maldonado, 2013; Martín, 2014), y en lo que se refiere a la realidad aumentada, progresivamente van apareciendo estudios reflexivos y conceptuales e investigaciones para abordar la problemática de cómo incorporar al terreno educativo (Cabero y García, 2016a; Villaustre y del Moral, 2016; Marín, 2016; Barroso y Gallego, 2017; Garay, Tejada, y Maiz, 2017). 
En este artículo pretendemos realizar un acercamiento conceptual a ambos entornos tecnológicos, y para ello presentaremos sus características distintivas, los puntos de encuentro entre ambas, y finalizaremos exponiendo algunos objetos realizados dentro de un proyecto de investigación.

\section{QUÉ ES B-LEARNING Y CUÁLES PUEDEN SER SUS CARACTERÍSTICAS DISTINTIVAS}

El b-learning, o aprendizaje híbrido o mezclado, como también se le conoce, es considerado como una evolución del e-learning, y suele llegar a definírsele como la realización de acciones formativas donde se combina la formación virtual con la presencial (Llorente y Cabero, 2009; Cubides y Martín, 2014; Salinas, Darder y De Benito, 2015). Más concretamente Salinas, Darder y De Benito, (2015, p. 157) lo entienden como:

un programa de educación formal en el que estudiante aprende, en parte, a través del aprendizaje en línea (con algún elemento de control del estudiante a través del tiempo, el lugar, la ruta y/o el ritmo) y en parte, en un lugar físico del campus con algún tipo de supervisión, y que estas distintas modalidades a lo largo del itinerario de aprendizaje en un curso o materia se interconectan para proporcionar una experiencia de aprendizaje integrado.

Acción formativa que, de acuerdo con diferentes autores (Llorente y Cabero, 2009; Cubides y Martín, 2014; Salinas, Darder y De Benito, 2015), aporta una serie de ventajas sintetizadas en la tabla 1. 
Tabla 1. Posibilidades del b-learning para estudiantes, docentes e instituciones

\begin{tabular}{|c|c|c|}
\hline Estudiantes & Docentes & Institución \\
\hline $\begin{array}{l}\text { Mejora los resultados } \\
\text { académicos. } \\
\text { Facilita la interacción } \\
\text { estudiante-docente y } \\
\text { estudiante-estudiante. } \\
\text { Aumenta la motivación y el } \\
\text { grado de satisfacción de los } \\
\text { estudiantes por la formación } \\
\text { en general y la formación } \\
\text { virtual en particular. } \\
\text { Se producen menos } \\
\text { abandonos de las acciones } \\
\text { formativas. } \\
\text { Mejora el autoconcepto } \\
\text { del alumno en la acción } \\
\text { formativa. }\end{array}$ & \begin{tabular}{|l|} 
Mejora la interacción \\
docente-estudiante. \\
El docente se encuentra \\
más cómodo y confiado en \\
la eficacia de la formación \\
virtual. \\
Facilita la superación del \\
nerviosismo tecnológico \\
del docente y el miedo al no \\
tener en la acción formativa a \\
nadie enfrente. \\
Mejora las competencias \\
digitales de los docentes. \\
Facilita la relación de \\
acciones formativas \\
colaborativas con los \\
estudiantes. \\
Facilita la planificación \\
educativa. \\
Favorece la realización de \\
acciones constructivistas de \\
la enseñanza.
\end{tabular} & $\begin{array}{l}\text { Aumento del número de } \\
\text { egresados. } \\
\text { Aumento del número de } \\
\text { alumnos matriculados en las } \\
\text { acciones formativas. } \\
\text { Reduce costes de la } \\
\text { formación presencial. } \\
\text { Mayor flexibilidad para } \\
\text { el diseño de materiales } \\
\text { educativos. } \\
\text { Propicia un diseño de } \\
\text { materiales más adaptado } \\
\text { a las características de los } \\
\text { estudiantes. }\end{array}$ \\
\hline
\end{tabular}

Pero hablar de b-learning es no perder de vista (Heik, 2012; Graham, Henrie y Gibbons, 2014; Salinas, Darder y De Benito, 2015), que se puede llevar a cabo desde diferentes perspectivas y posiciones, que van desde la rotación en cada una de las diversas acciones formativas entre lo virtual y lo presencial, hasta las situaciones flexibles, en las cuales es el estudiante el que toma la decisión de cambiar entre lo virtual y lo presencial, aunque autores como Heick (2012), amplían dichas opciones con las de laboratorios on-line (los contenidos se distribuyen íntegramente en una plataforma, pero en escenarios presenciales donde los estudiantes deben realizar diferentes tipos de prácticas), auto-blended (el alumnado, según sus propios intereses, realiza una acción formativa de manera virtual) y distribución on-line (los contenidos son ofertados por una plataforma y por el docente, trabajando los profesores a distancia aunque las acciones presenciales son siempre obligatorias). De todas formas, como indican Turbo y Hernández (2014, p. 105), no debemos perder de vista que el b-learning

no implica, o no debe implicar, un resultado o solo una suma sea ésta de métodos o de tecnologías. El BL implica un resultado con valor añadido, donde existe coherencia en la combinación, y donde unos métodos o tecnologías usadas en la presencialidad se adaptan o integran perfectamente con lo planificado y desarrollado en la virtualidad. 
En cierta medida hablar de b-learning es hacerlo desde una nueva escenografía comunicativa para la formación, donde a las ventajas de la presencialidad se le incorporan las de la virtualidad, y a la ruptura del espacio y el tiempo, se le incorpore la concreción de los mismos. Por otra parte, señalar que el b-learning se constituye no solo como una acción formativa que armoniza lo virtual y lo presencial, sino más bien como un ecosistema de formación, donde se combinan estrategias de enseñanzas, metodologías de aprendizaje y tecnologías. Este ecosistema que se enriquecerá en el futuro con los dispositivos móviles, propiciando con ello el aprendizaje ubicuo (Vázquez-Cano y Sevillano, 2015), y la incorporación de tecnologías como la RA, a la cual nos referiremos a continuación.

\section{QUÉ ES LA REALIDAD AUMENTADA Y CUÁLES SON SUS CARACTERÍSTICAS DISTINTIVAS}

La Realidad Aumentada (RA) es una de las tecnologías que está teniendo una gran penetración en diferentes sectores, que van desde el de ocio, al publicitario, al militar, al de turismo y al sector de la formación (Prendes, 2015; Olesky y Wnuk, 2016; Manuri y Sanna, 2016; Marín, 2016; Schmalstieg y Höllerer, 2016).

Hablar de RA es referirnos a una tecnología que persigue la combinación en tiempo real, -con la participación directa del usuario-, de información digital y real a través de diversos dispositivos tecnológicos, que van desde las tablet, las webcam, los smartphone o las gafas, estando esta tecnología a medio camino entre la realidad y los entornos virtuales inmersivos, construyéndose en su combinación una nueva realidad donde la tecnología aporta información complementaria a la existente en la realidad (Baldiris et al., 2016; Cabero y Barroso; 2016a, 2016b; Cabero y García, 2016; Cabero, Leiva, Moreno, Barroso y López, 2016; Barroso y Gallego, 2017a, 2017b).

Frente a otras tecnologías, la RA presenta una serie de características distintivas: es una tecnología mixta de combinación de lo real y lo virtual, la interacción de lo real y virtual se produce en tiempo real, requiere de la participación del usuario que se produce a diferentes niveles, permite la combinación de información en distintos sistemas simbólicos y recursos, que van desde los clip de vídeos, los podcast de audio, documentos en formato PDF, espacios Web, animaciones en 3D, simulaciones, etc. y crear con ello diferentes capas informativas sobre el objeto real (Di Serio, Ibáñez y Delgado, 2013; Cabero y García, 2016a; Schmalstieg y Höllerer, 2016).

Para la formación ofrece distintas posibilidades y presenta también una serie de dificultades, como han sugerido distintos autores (Bacca, Baldiris, Fabregat, Graf y Kinshuk, 2014; Barba, Yasaca y Manosalvas, 2015; Jamali, Fairuz, Wai y Oskam, 2015; Fonseca, Redondo y Valls, 2015; Han, Jo, Hyun y So, 2015; Prendes, 2015; Marín, 2016, 2017; Reinoso, 2016; Santos et al., 2016), y que se han sintetizado en la tabla 2. 
Tabla 2. Posibilidades y dificultades para la incorporación educativa de la RA

\begin{tabular}{|c|c|}
\hline $\begin{array}{l}\text { Posibilidades Educativas de la } \\
\text { Realidad Aumentada }\end{array}$ & Dificultades para su incorporación \\
\hline $\begin{array}{l}\text { Enriquece la información de la realidad para } \\
\text { hacerla más comprensible al estudiante. } \\
\text { Crea entornos formativos multimedias. } \\
\text { Potencia el aprendizaje ubicuo y móvil. } \\
\text { Facilita eliminar información superflua } \\
\text { que pueda dificultar la observación de la } \\
\text { información importante. } \\
\text { Permite crear laboratorios o simuladores } \\
\text { seguros para los estudiantes. } \\
\text { Puede convertir a los alumnos en } \\
\text { "proconsumidores" de objetos de } \\
\text { aprendizaje en formato RA. } \\
\text { Potencia el enriquecer documentos escritos } \\
\text { con información complementaria en clip de } \\
\text { vídeos o podcast de audio. } \\
\text { Facilita el desarrollo de una formación } \\
\text { activa. } \\
\text { Crea entornos lúdicos y motivantes para la } \\
\text { formación. } \\
\text { Permite el visionado y la observación de un } \\
\text { objeto desde múltiples perspectivas, que son } \\
\text { seleccionadas por el discente. } \\
\text { Y los objetos creados pueden ser utilizados } \\
\text { en diferentes metodologías y estrategias de } \\
\text { enseñanza. }\end{array}$ & $\begin{array}{l}\text { Falta de investigaciones. } \\
\text { La novedad de la tecnología que requiere } \\
\text { unas mínimas competencias tecnológicas } \\
\text { para el docente y discente. } \\
\text { La novedad de la tecnología. } \\
\text { La falta de objetos de aprendizaje para su } \\
\text { incorporación a situaciones de enseñanza. } \\
\text { La disociación cognitiva que produce el } \\
\text { interaccionar en un contexto que mezcla lo } \\
\text { real y lo virtual. } \\
\text { La formación del docente para su } \\
\text { incorporación educativa. } \\
\text { No disponer de un marco conceptual } \\
\text { consolidado para su incorporación. } \\
\text { Que es poco conocida para los docentes. } \\
\text { Y la rapidez de cómo está evolucionando. }\end{array}$ \\
\hline
\end{tabular}

Su incorporación a la enseñanza se está llevando a cabo en diferentes niveles educativos: educación infantil y primaria (Prendes, 2015; Gómez, 2016; Villalustre y Del Moral, 2016; Cozar-Gutiérrez y Sáez-López, 2017), secundaria-bachilleratoformación profesional (Kamarainen, Metcalf, Grotzer, Browne, Mazzuca, Tutwiler y Dede, 2013; Cabero, Leiva, Moreno, Barroso y López, 2016; Gómez, 2016) y universitario (Lin, Duh, Li, Wang y Tsai, 2013; Rodríguez, 2013; Cabero y García, 2016b; Barroso y Gallego, 2017; Fernández, 2017); y en distintas áreas curriculares: ingeniería (De la Torre et al., 2013), arquitectura (Redondo et al., 2012; De la Torre et al., 2013), formación del profesorado y ciencias de la educación (Fernández, 2017; Garay, Tejada, y Castaño, 2017; Garay, Tejada y Maiz, 2017), urbanismo (Carozza, Tingdahl y Gool, 2014), matemáticas-geometría (Coimbra, Cardoso y Mateus, 2015), arte e historia (Ruiz, 2011), idiomas (Sánchez, 2017), tecnología (Rodríguez, 2013), Diseño (Ko, Chang, Chen y Hua, 2011), y física y química (Pasaréti et al., 2011; Lin, Duh, Li, Wang y Tsai, 2013). 
De todas formas, en su incorporación debe tenerse en cuenta lo señalado por Cuendet, Bonnard, Son Do-Lenh y Dillenbourg (2013), cuando llaman la atención respecto a que en su utilización en la formación se deben contemplar dos aspectos: 1) que el sistema propuesto debería ser lo suficientemente flexible para que el profesor lo adapte a las necesidades de sus estudiantes y 2) que el sistema deberá tener en consideración las restricciones presentes en el contexto educativo al cual se aplica. Así como que los objetos de aprendizaje que se construyan deben poseer una serie de peculiaridades: que sean breves y concretos, que posean una concepción multimedia, han de ser flexibles para incorporarlos a diferentes propuestas formativas, y adaptados a las diversas características de los distintos dispositivos tecnológicos donde pueden ser observados.

Como ya señalamos uno de los grandes inconvenientes para su incorporación a la formación es la falta de investigaciones, aunque debemos reconocer que en los últimos tiempos su producción científica ha aumentado considerablemente (Fombona y Pascual, 2017), y empiezan a abordarse proyectos ambiciosos como RAFODIUN (Realidad Aumentada para Aumentar la Formación. Diseño, Producción y Evaluación de Programas de Realidad Aumentada para la Formación Universitaria (EDU2014-57446-P), http://intra.sav.us.es/proyectorafodiun/), centrado en analizar sus posibilidades educativas en la formación universitaria.

\section{B-LEARNING Y REALIDAD AUMENTADA: EXPERIENCIAS DE DISEÑO DOCENTE}

Hablar de b-learning y RA es no perder de vista desde el comienzo, como se señaló anteriormente, que el b-learning se configura como un ecosistema de formación virtual, donde pueden entrar en juego diferentes tecnologías, que van desde las plataformas de teleformación, las herramientas de la Web 2.o, hasta las denominadas tecnologías emergentes; entre las cuales se sitúa la RA, y también distintas metodologías que pueden ir desde el aprendizaje basado en problemas, hasta el estudio de casos, y el aprendizaje colaborativo. Todas estas metodologías se están movilizando en la RA (Wojciechowski y Cellary, 2013; Lukosch, Billinghurst, Alem y Kiyokawa, 2015).

Por otra parte, la combinación de acciones formativas en b-learning y RA puede favorecer claramente un aprendizaje activo para el estudiante, pues como apuntan Beetham y Sharpe (2013), este tipo de aprendizaje se ve favorecido por la combinación de tres elementos: espacio, pedagogía y tecnología (figura 1). Estos elementos claramente se alcanzan con la combinación de las dos propuestas tecnológicas comentadas, pues por una parte se facilita trabajar en distintos espacios y escenarios, tanto virtuales como reales y tanto presenciales como a distancia; se movilizan diferentes elementos/recursos digitales que propician la creación de variados y diversos escenarios tecnológicos, y por otra, se dispone de una fundamentación 
teórica, aunque es cierto que resulta más contundente en las acciones b-learning que las sustentadas en la RA.

Figura 1. Combinación de elementos para alcanzar un aprendizaje activo

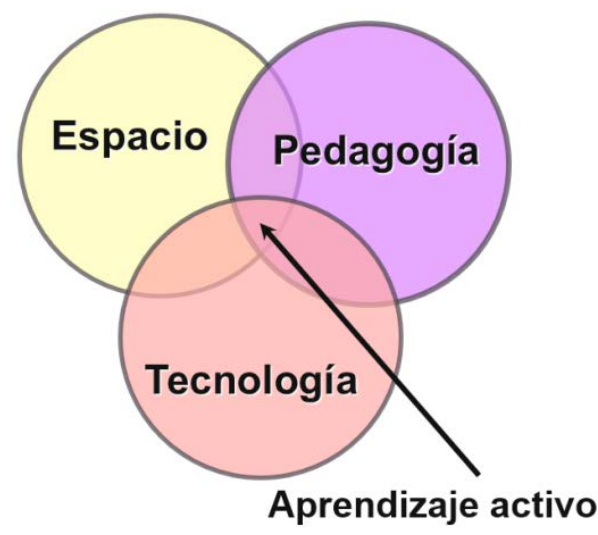

Fuente: Beetham y Sharpe (2013)

Desde un punto de vista tecnológico, la RA puede ser de utilidad en acciones formativas de $b$-learning desde diferentes aproximaciones: a) el enriquecimiento de los apuntes y materiales ofrecidos a los estudiantes con documentos audiovisuales adicionales, b) la utilización de simuladores y laboratorios virtuales, c) la creación y presentación de objetos para su manipulación por parte del estudiante y la observación de los mismos desde diferentes perspectivas, d) la elaboración de materiales para la realización de prácticas por parte de los estudiantes, y e) la concreción y contextualización de la información presentada. Todos ellos sin olvidar que la RA facilita la concreción de los conceptos abstractos.

De algunos de ellos hemos efectuado diferentes investigaciones dentro del proyecto RAFODIUN, y vamos a ofrecer algunos de los resultados encontrados. El enriquecimiento de apuntes se refiere a la utilización de la RA para incorporar en documentos Word o PDF, diferentes recursos (clip de vídeos, podcast de audio, etc.). Los materiales que se realizaron para el estudio trataban las temáticas de los "roles de utilización del vídeo" y la "construcción de guías didácticas de acompañamiento". Para la producción de los diferentes objetos de aprendizaje, se utilizaron diferentes programas que presentamos en la tabla 3 . 
Tabla 3. Software utilizado producción de objetos

\begin{tabular}{|l|l|}
\hline \multicolumn{1}{|c|}{ SOFTWARE } & \multicolumn{1}{c|}{ PARA QUÉ SE UTILIZA } \\
\hline Metaio Creator & Programación realidad aumentada. \\
\hline Metaio SDK & Kit de desarrollo de software RA. \\
\hline Eclipse & Entorno de desarrollo Java. Exportación apk para android. \\
\hline Xcode & $\begin{array}{l}\text { Entorno de desarrollo Java. Exportación .ipa para IOS.Subida a la } \\
\text { app store. }\end{array}$ \\
\hline Adobe After effects & Postproducción de vídeo y sonido. Chroma, Rotobrush, Key Light. \\
\hline Adobe Photoshop & $\begin{array}{l}\text { Postproducción de imagen. Grafismos. Photomerge. Texturizado } \\
\text { 3D. }\end{array}$ \\
\hline Macromedia Fireworks & Postproducción de imagen. Grafismos. \\
\hline Ffmpeg & $\begin{array}{l}\text { Programación sobre el codec para exportación vídeos 3g2. } \\
\text { Augment----RA. }\end{array}$ \\
\hline Notepad ++ & Editor profesional de texto para retoque de código. \\
\hline Astrum & Creador de instalador windows. \\
\hline
\end{tabular}

En la figura 2, puede observarse la forma en la cual se le presentaban los apuntes, donde además de la información y los lanzadores de clip de vídeo mediante RA, se ofrecía información respecto a cómo debía instalarse la App correspondiente, bibliografía de la temática y referencias para saber más. Estos apuntes, como otros, pueden descargarse de la siguiente dirección Web: http://intra.sav.us.es/ proyectorafodiun/index.php/objetos-en-ra

Figura 2. Apuntes elaborados
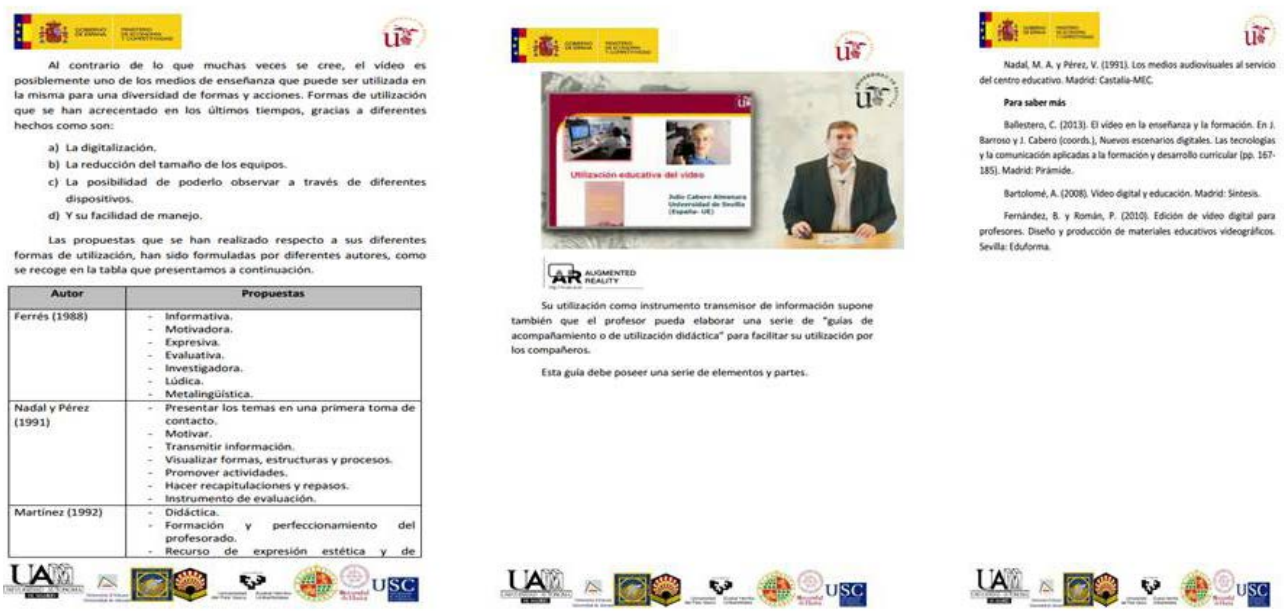
La investigación se llevó a cabo con estudiantes que cursaban las asignaturas de “Tecnología Educativa” de segundo curso del grado de Pedagogía impartido en la Facultad de Ciencias de la Educación de la Universidad de Sevilla, estando la muestra conformada por 198 estudiantes (46 hombres-22,42\%- y 152 mujeres-75,58\%).

Para su incorporación a la enseñanza y la realización de la investigación se siguieron 4 etapas: en una sesión previa se les aplicó el pretest, en una segunda sesión se les explicó a los alumnos el funcionamiento de los objetos y se realizaron diferentes comentarios sobre la utilización de la RA, y el lugar desde el cual deberían descargarse la app para instalarla en sus dispositivos móviles y los apuntes, la tercera duró un período de dos semanas en las cuales los estudiantes trabajaron de forma individual con los objetos y en las clases de prácticas de la asignatura se les resolvieron las dudas que se les fueron presentando, bien sobre contenidos expuestos, sobre la RA o sobre la manipulación de los objetos; y en una última fase en la cual se cumplimentaron los instrumentos de diagnóstico de la motivación, el rendimiento y la calidad del objeto producido. Cabe señalar que los alumnos sabían que las calificaciones alcanzadas en la experiencia se tendrían en cuenta para las calificaciones de la asignatura. Podemos concretar los resultados encontrados en los siguientes aspectos:

- Los alumnos mostraron un alto grado de aceptación de la tecnología de la RA, medida a través del TAM ("Technology Acceptance Model") formulado por Davis (1989).

- Hubo diferencias estadísticas significativas entre el rendimiento adquirido por los estudiantes entre las puntuaciones de pretest y postest.

- Los alumnos se sintieron motivados al participar en la experiencia, diagnosticada la motivación a través del "Instructional Material Motivational Survey" (IMMS) de Keller (2010).

- Los alumnos valoraron la calidad técnica y educativa de los objetos producidos en una escala de 1 a 5, con una media de 3,66 para el del vídeo y del 3,82 para el de diseño.

- No se encontraron diferencias significativas en rendimiento, motivación, grado de aceptación y valoración de los objetos, entre los dos objetos producidos.

- No existieron diferencias significativas en los resultados encontrados entre los hombres y las mujeres.

Otra de las formas de combinar las tecnologías de las que estamos hablando, consiste en mezclarlas de tal forma, que a través del b-learning y la plataforma que se utilice para ello, se le presenta los contenidos y las prácticas que se deben realizar, utilizándose la RA para ofrecer laboratorios y simuladores que faciliten la realización de las prácticas. Ejemplo de esta combinación nos la encontramos en el trabajo realizado por El Kabtane, El Adnani, Sadgal y Mourdi (2016), quienes en su estudio ofrecen una combinación para solventar la falta de actividades prácticas 
en la plataforma de formación virtual, facilitando de esta forma una interacción con objetos virtuales que permiten realizar las actividades prácticas por parte de los estudiantes, lo que reduce los riesgos de sobrecarga, falta de materiales y el encarecimiento de la acción formativa.

La incorporación de objetos de RA puede ser de gran utilidad para que los estudiantes puedan manipular diferentes objetos y observarlos desde distintas perspectivas. Claro ejemplo de lo que nos referimos, son las experiencias realizadas a través del proyecto RAFODIUN con alumnos de medicina, donde a los estudiantes se le presentan diferentes órganos y huesos, sobre los cuales puedan realizar distintas actividades, como son: rotarlos para observarlos desde diferentes perspectivas, ampliarlos, o cambiar el punto de vista de observación (Barroso, Cabero y Moreno, 2016; Barroso y Cabero, 2016) (figura 3).

Figura 3. Objetos en RA producidos para la formación en medicina

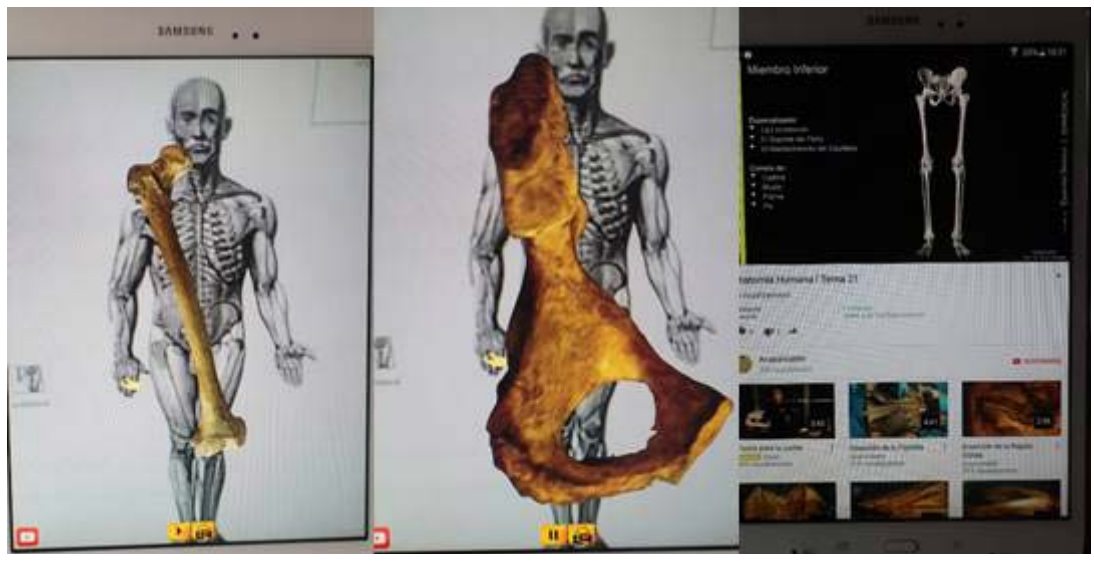

La evaluación de estos objetos por los estudiantes se llevó a cabo en un estudio piloto con alumnos del Grado de Medicina que cursaban la asignatura de "Anatomía Humana I" de primer curso y que es impartida por el Departamento de "Anatomía y Embriología Humana". El número de participantes fue de 50 (21 hombres $-42 \%-$ y 29 mujeres $-58 \%$ ).

La evaluación de los materiales se llevó a cabo en una sesión donde se realizaron las siguientes acciones: a) explicación de en qué consistía la RA; b) demostración de los diferentes objetos producidos; c) presentación del lugar del cual podrían bajarse las distintas guías de los cuatro objetos que se le iban a presentar y que posteriormente deberían evaluar (shoulder, coxofemoral, cervical y ankle) (http:// intra.sav.us.es/proyectorafodiun/index.php/objetos-en-ra); d) trabajo individual de los alumnos con sus dispositivos móviles para interaccionar con los diferentes 
objetos presentados; y e) cumplimentación de los cuestionarios de evaluación de los objetos de aprendizajes presentados por parte de los estudiantes.

Indicar que, en una escala de 1 a 5 , las valoraciones medias que efectuaron los estudiantes de los diferentes objetos producidos, fueron verdaderamente significativas, como pueden observarse en la tabla 4 .

Tabla 4. Valoraciones de los objetos

\begin{tabular}{|l|c|c|}
\hline \multicolumn{1}{|c|}{ Objeto } & Media & D.t \\
\hline Todos los objetos & 4,33 & 0,44 \\
\hline Objeto Shoulder & 4,43 & 0,58 \\
\hline Objeto Coxofemoral & 4,36 & 0,49 \\
\hline Objeto Cervical & 4,29 & 0,44 \\
\hline Objeto Ankle & 4,23 & 0,40 \\
\hline
\end{tabular}

Señalar que también en la experiencia desarrollada, los alumnos mostraron un alto grado de aceptación de esta tecnología, medida también a través del TAM (Davis, 1989). Al mismo tiempo tampoco se encontraron diferencias significativas entre el género de los estudiantes.

Las experiencias realizadas nos han permitido alcanzar una serie de conclusiones a contemplar a la hora del diseño de objetos de aprendizaje en RA, como son:

- Hacer módulos de contenidos breves y directos, que no propicien un desbordamiento y desorientación cognitiva en el estudiante en el desplazamiento por el objeto.

- Tener en cuenta, en su diseño, los principios de flexibilidad y simplicidad, para evitar que el alumno se desoriente en la utilización del objeto.

- Que sea accesible y tolerante a los errores, de manera que la interacción con el objeto sea intuitiva, cómoda y fácil para el estudiante.

- Tendencia a la producción de objetos multimedia; es decir, que incorporen elementos de diferentes sistemas simbólicos y que por tanto, puedan activar los diferentes tipos de inteligentes.

- Propiciar la interacción del sujeto con el objeto, y por tanto, que tenga una construcción hacia la acción.

- Poseer una construcción multiplataforma.

La utilización de objetos de RA en acciones de b-learning, pueden ser de utilidad para la realización de prácticas y la ejercitación sobre los contenidos presentados. Y en este aspecto Cubillo et al. (2014, p. 245) reseñan que la RA puede ser beneficiosa para realizar experimentos o prácticas difíciles de realizar por el coste de los instrumentos, la disponibilidad de las instalaciones ya sea por espacio y/o por tiempo, la dificultad 
de realizar experimentos complejos y peligrosos, y el poder observar los resultados de experimentos de larga duración reducidos a minutos y segundos. Esta ejercitación se está llevando a cabo en diferentes áreas como las Matemáticas (Coimbra, Cardoso y Mateus, 2015) o la Arquitectura (Redondo, Sánchez y Moya, 2012); propiciando que el estudiante pueda observar la traslación de los objetos y las consecuencias de la variación de determinadas variables sobre la realidad.

Nuestras últimas citas se van a referir a la concreción y contextualización de la información presentada por la plataforma, hecho que progresivamente va a ser más significativo, teniendo en cuenta la potencia que para la geolocalización, están adquiriendo los dispositivos móviles, lo que facilitaría la interpretación de mapas geográficos (Carbonnel y Bermejo, 2017).

Como ya se ha señalado anteriormente las investigaciones sobre la RA no han sido muy numerosas, pero si es cierto que las que se están llevando a cabo van aportando un volumen de resultados que nos llevan a señalar que la utilización de la RA, eso sí dentro de una propuesta pedagógica razonada y justificada, facilita la creación de entornos educativos de calidad. Así, diferentes estudios han puesto de manifiesto que, en su utilización, los estudiantes muestran actitudes favorables hacia ella y un elevado grado de satisfacción (Han et al., 2015; Cabero, García y Arroyo, 2016; Kim, Hwang y Zo, 2016), que su utilización aumenta la motivación que despliegan los estudiantes hacia el aprendizaje (Kamarainen, et al., 2013; Di Serio, et al., 2013; Cózar et al., 2015; Wei, Weng, Liu y Wang, 2015), que favorecen la creación de un contexto activo y constructivista para el aprendizaje (Fombona, Pascual, y Madeira, 2012; Wojciechowski y Cellary, 2013), y que su utilización mejora los resultados de aprendizajes (Bongiovani, 2013; Chang, Wu y Hsu, 2013; Kamarainen et al., 2013).

Para finalizar nuestro análisis, indicar que estamos de acuerdo con lo expresado por Wei et al., (2015) cuando señalan que la incorporación de la RA a la formación pasa necesariamente por que el docente diseñe la acción formativa de manera creativa, a lo que nosotros añadiríamos a que se busquen marcos conceptuales desde donde justificar la integración del b-learning y la RA. Y de esta forma estamos seguros que en un futuro próximo, los formadores y educadores podrán emplear la RA para crear escenarios cotidianos de capacitación más realistas y aplicables que puedan combinarse con la formación virtual. Al mismo tiempo, y teniendo en cuenta que la mayoría de las personas aprenden mucho mejor cuando observan y hacen, en lugar de exclusivamente leyendo, la incorporación de objetos en RA puede proporcionar la creación de escenarios más reales y, con ello, generar ambientes enriquecidos, seguros y controlados.

\section{Agradecimiento}

El trabajo se enmarca dentro de un proyecto de investigación I+D financiado por el Ministerio de Economía y Competitividad del Gobierno de España denominado: "Realidad aumentada para aumentar la formación, diseño, producción y evaluación 
de programas de realidad aumentada para la formación universitaria" (EDU-5746-P - Proyecto Rafodiun).

\section{REFERENCIAS BIBLIOGRÁFICAS}

Bacca, J., Baldiris, S., Fabregat, R., Graf, S., y Kinshuk, G. (2014). Augmented Reality Trends in Education: A Systematic Review of Research and Applications. Educational Technology y Society, 17(4), 133-149.

Baldiris, S., Duque, N., Salas, D., Bernal, J., Fabregat, R., Mendoza, R., Puerta, Y., Puello, J., Solano, I., y Martínez, L. (2016). Recursos Educativos Aumentados. Una oportunidad para la inclusión. Colombia: Sello Editorial Tecnolkógico Comfenalco.

Barba, R., Yasaca, S., y Manosalvas, C. (2015). Impacto de la realidad aumentada móvil en el proceso enseñanza-aprendizaje de estudiantes universitarios del área de medicina. AIDIPE, Investigar con y para la Sociedad, 3, (pp. 1421-1429). Cádiz: Bubok Publishing S.L.

Barroso, J., y Cabero, J. (2016). Evaluación de objetos de aprendizaje en realidad aumentada: estudio piloto en el grado de medicina. Enseñanza \& Teaching, 34, 149-167. doi: https://doi.org/10.14201/ et2016342149167

Barroso, J., y Gallego, O. (2017a). La realidad aumentada y su aplicación en la educación superior. Revista del Salomé, 1(29), 111124.

Barroso, J., y Gallego, O. (2017b). Producción de recursos de aprendizaje apoyados en Realidad Aumentada por parte de los estudiantes de magisterio. EDMETIC, Revista de Educación Mediática y TIC, 6(1), 23-38.

Barroso, J., Cabero, J., y Moreno, A. (2016). La utilización de objetos de aprendizaje en realidad aumentada en la enseñanza de la medicina. Innoeduca. International Journal of Technology and Educational Innovation, 2(2), 77-83. doi: http:/ dx.doi.org/10.20548/innoeduca.2016. v2i2.1955

Beetham, H., y Sharpe, R. (Eds.) (2013). Rethinking Pedagogy for a Digital Age: Designing for 21st Century Learning. New York: Routleedge.

Cabero, J., y Barroso, J. (2016a). The educational possibilities of Augmented Reality. NAER. New Approaches in Educational Research, 5(1), 44-50. doi: 10.7821/naer.2016.1.140.

Cabero, J., y Barroso, J. (2016b). Ecosistema de aprendizaje de "realidad aumentada": posibilidades educativas. Tecnología, Ciencia y Educación, 5, 141-154.

Cabero, J., y García, F. (Coords.) (2016). Realidad Aumentada. Tecnología para la formación. Madrid: Síntesis.

Cabero, J., García, F., y Arroyo, C. (2016). La producción de objetos de aprendizaje en realidad aumentada para la formación universitaria en le SAV de la Universidad de Sevilla. En L. Villalustre y M. E. Del Moral (Coords.), Experiencias interactivas con realidad aumentada en las aulas (pp9-30). Barcelona: Octaedro.

Cabero, J., Leiva, J., Moreno, N., Barroso, J., y López, E. (2016). Realidad Aumentada y educación. Barcelona: Octaedro.

Carbonnel, C., y Bermejo, L. (2017). Landscape interpretation with augmented reality and maps to improve spatial orientation skill. Journal of Geography in Higher Education, 41, 119-133. doi: http://dx.doi.org/10.1080/03098265.20 16.1260530

Carozza, L., Tingdahi, D., Bosché, F., y Gool, L. (2014). Markerless Vision-Based Augmented Reality for Urban Planning. Computer-Aided Civil and Infrastructure Engineering, oo, 1-16. 
Chang, H., Wu, K., y Hsu, Y. (2013). Integrating a mobile augmented reality activity to contextualize student learning of a socioscientific issue. British Journal of Educational Technology, 44(3), E95-E99. doi: 10.1111/j.1467-8535.2012.01379.x.

Coimbra, M., Cardoso, T., y Mateus, A. (2015). Augmented Reality: an Enhancer for Higher Education Students in Math's learning? Procedia Computer Science, 67, 332-339. doi 10.1016/j.procs.2015.09.277.

Cozar-Gutiérrez, R., y Sáez-López, J. M. (2017). Realidad aumentada, proyectos en el aula de primaria: experiencias y casos en Ciencias Sociales. EDMETIC. Revista de Educación Mediática y TIC, 6(1), 165180.

Cubides, N., y Martín, A.V. (2014). Los modelos formativos combinados b-Learning: perspectivas para la enseñanza universitaria. En A.V. Martín, Blended learning en Educación Superior, (75-100). Madrid: Síntesis.

Davis, F. (1989), Perceived usefulness, perceived ease of use, and user acceptance of information technology, MIS Quarterly, 13(3), 319-340, doi: 10.2307/249008.

De la Torre, J., Martin-Dorta, N., Saorín, J.L., Carbonell, C., y Contero, M. (2013). Entorno de aprendizaje ubicuo con realidad aumentada y tabletas para estimular la comprensión del espacio tridimensional. RED, Revista de Educación a Distancia, 37. Recuperado de http://www.um.es/ead/red/37

De Pedro, J., y Méndez, C. L. M. (2012). Realidad Aumentada: Una Alternativa Metodológica en la Educación Primaria Nicaragüense. IEEE-RITA, 7, 102-108.

El Kabtane, H., El Adnani, M., Sadgal, M., y Mourdi, Y. (2016). An Augmented Reality Approach to Integrate Practical Activities in E-Learning Systems. International Journal of Advanced Computer Science and Applications (IJACSA), 7(2), 107-117.

Fernández, B. (2017). Factores que influyen en el uso y aceptación de objetos de aprendizaje de realidad aumentada en estudios universitarios de Educación Primaria. EDMETIC. Revista de Educación Mediática y TIC, 6(1), 200220.

Fombona, J., y Pascual, M. A. (2017). La producción científica sobre Realidad Aumentada, un análisis de la situación educativa desde la perspectiva SCOPUS. EDMETIC. Revista de Educación Mediática y TIC, 6(1), 39-61.

Fombona, J., Pascual, M. J., y Madeira, M. F. (2012). Realidad aumentada, una evolución de las aplicaciones de los dispositivos móviles. Píxel-Bit. Revista de Medios y Educación, 41, 197-210.

Fonseca, D., Redondo, E., y Valls, F. (2016): Motivación y mejora académica utilizando realidad aumentada para el estudio de modelos tridimensionales arquitectónicos. Education in the Knowledge Society, EKS, 17(1), 45-64.

Garay, U., Tejada, E., y Castaño, C. (2017). Percepciones del alumnado hacia el aprendizaje mediante objetos educativos enriquecidos con realidad aumentada. EDMETIC. Revista de Educación Mediática y TIC, 6(1), 145-164.

Garay, U., Tejada, E., y Maiz, I. (2017). Valoración de objetos educativos enriquecidos con realidad aumentada: una experiencia con alumnado de máster universitario. Pixel-Bit. Revista de Medios y Educación, 5O, 19-31. doi:http://dx.doi. org/10.12795/pixelbit.2017.i50.01

Gómez, M. (2016). Aplicaciones educativas de la realidad aumentada en contextos no universitarios. En J. Cabero y F. García, (Coords.), Realidad Aumentada. Tecnología para la formación (113-125). Madrid: Síntesis.

Graham, C., Henrie, C., y Gibbons, A. (2014). Developing models and theory for blended learning research. En A. Picciano, C. Dziuban y C. Graham (Eds.), Blended learning: research perspectives, 2, (1333). Nueva York: Routledge. 
Han, J. Jo, M., Hyun, E., y So, H. (2015). Examining young children's perception toward augmented reality-infused dramatic play. Education Technology Research Development, 63, 455-474.

Jamali, S., Fairuz, M. Wai, K., y Oskam, Ch. (2015): Utilising mobile-augmented reality for learning human anatomy. Procedia-Social and Behavioral Sciences, 197, 659-668. doi: http://dx.doi. org/10.1016/j.sbspro.2015.07.054

Johnson, L., Adams, S., Cummins, M., Estrada, V., Freeman, A., y Hall, C. (2016). NMC Horizon Report: 2016 Higher Education Edition. Austin, Texas: The New Media Consortium.

Johnson, L., Adams Becker, S., Cummins, M., Estrada, V., Freeman, A., y Ludgate, H. (2013). Technology Outlook for Australian Tertiary Education 20132018: An NMC Horizon Project Regional Analysis. Austin, Texas: The New Media Consortium.

Kamarainen, A., Metcalf, Sh., Grotzer, T., Browne, A., Mazzuca, D., Tutwiler, M., y Dede, Ch. (2013). EcoMOBILE: Integrating augmented reality and probeware with environmental education field trips. Computers \& Education, 68, 545-556. doi: 10.1016/j. compedu.2013.02.018.

Keller, J. M. (2010). Motivational design for learning and performance. New York: Springer Science+Business.

Kim, K., Hwang, J., y Zo, H. (2016). Understanding users' continuance intention toward smartphone augmented reality applications. Information Development, 32(2) 161-174. doi: 10.1177/0266666914535119.

Ko, Ch-H., Chang, T.C, Chen, Y.-H., y Hua, L. A. (2011). The Application of Augmented Reality to Design Education. En M. Chang, Y. Hwang, M. P. Cheng y W. Muller (Eds.). Edutainment Technologies. Educational Games and VirtualReality/Augmented
Reality Applications (20-24). Heidelberg Berlin: Springer.

Lin, T., Duh, H. B. L., Li, N., Wang, H.Y.,y Tsai, C. C. (2013). An investigation of learners' collaborative knowledge construction performances and behavior patterns in an augmented reality simulation system. Computers \& Education, 68, 314-321. doi: 10.1016/j.compedu.2013.05.011.

Lukosch, S., Billinghurst, M., Alem, L., y Kiyokawa, K. (2015). Collaboration in Augmented Reality. Computer Supported Cooperative Work (CSCW), 24, 515-525. doi: 10.1007/s10606-015-9239-0.

Llorente, M. C., y Cabero, J. (2009). La formación semipresencial a través de redes telemáticas (blended learning). Barcelona: Da Vinci.

Manuri, F., y Sanna, A. (2016). A Survey on Applications of Augmented Reality. ACSIJ Advances in Computer Science: an International Journal, 5(1), 19, 18-27.

Marín, V. (2016). Posibilidades de uso de la realidad aumentada en la educación inclusiva. Estudio de caso. Ensayos, Revista de la Facultad de Educación de Albacete, 31(2), 57-68.

Marín, V. (2017). The relationships between Augmented Reality and inclusive education in Higher Education. Bordón, 69(3). 125-142. doi:10.13042/ Bordon.2017.51123.

Martín, A.V. (2014). Blended learning en Educación Superior. Madrid: Síntesis.

NMC (2017). NMC Horizon Report Preview 2017 Higher Education Edition. Austin, Texas: The New Media Consortium.

Olesky, T., y Wnuk, A. (2016). Augmented places: An impact of embodied historical experience on attitudes towards places. Computers in Human Behavior, 57, 11-16. http://dx.doi.org/10.1016/i. chb.2015.12.014

Pasaréti, O., Hajdu, H., Matuszka, J., Jambori, A., Molnar, T., y Turcsányi-Szabó, M. (2011). Augmented Reality in education. INFODIDACT 2011 Informatika 
Szakmódszertani

Konferencia.

Recuperado de http://people.inf.elte.hu/ tomintt/infodidact 2011.pdf

Prendes, C. (2015). Realidad aumentada y educación: análisis de experiencias prácticas. Pixel-Bit. Revista de Medios y Educación, 46, 187-203. doi: 10.12795/ pixelbit.2015.146.12.

Redondo, E., Sánchez, A., y Moya, J. (2012). La ciudad como aula digital. Enseñando urbanismo y arquitectura mediante mobile learning y la realidad aumentada. Un estudio de viabilidad y de caso. Ace: Architecture, City and Environment, Z(19). Recuperado de http://upcommons. upc.edu/revistes/handle/2099/12344

Reinoso, R. (2016). Realidad aumentada posibilidades y usos educativos. En S. Baldiris (Ed.), Recursos Educativos Aumentados: Una oportunidad para la inclusión (8-29). Colombia: Sello Editorial Tecnológico Comfenalco.

Ruiz, D. (2011). La Realidad Aumentada y su dimensión en el arte: La obra aumentada. Arte y Políticas de Identidad, 5, 129-144.

Salinas, J., Darder, A., y De Benito, B. (2015). Las TIC en la enseñanza superior: e-learning, b-learning, y m-learning. En J. Cabero y J. Barroso (Coords.), Nuevos retos en tecnología educativa (153-173). Madrid: Síntesis.

Sánchez, J. (2017). El potencial de la realidad aumentada en la enseñanza de español como lengua extranjera. EDMETIC. Revista de Educación Mediática y TIC, 6(1), 62-80.

Santos, M. Wolde, A., Taketomi, T., Yamamoto, G., Rodrigo, M., Sandor, Ch., y Kato, H. (2016). Augmented reality as multimedia: the case for situated vocabulary learning. Research and Practice in Techology Enhanced Learning, 11(4), 1-23.

Schmalstieg, D., y Höllerer, T. (2016). Augmented reality: principles and practice. Boston: Addison-Wesley.

Sevillano, M. L. (2015). El contexto socioeducativo de la ubicuidad y movilidad. En E. Vázquez-Cano y M. L. Sevillano (Eds.), Dispositivos digitales móviles en educación. El aprendizaje ubicuo (17-7). Madrid: Narcea.

Tecnológico de Monterrey (2015). Reporte EduTrends. Radar de Innovación Educativa 2015. Monterrey: Tecnológico de Monterrey.

Turpo, O., y Hernández, M. J. (2014). La convergencia pedagógica y tecnológica de la modalidad Blended Learning. En A. V. Martín, Blended learning en Educación Superior, (101-119). Madrid: Síntesis.

Vázquez-Cano, E., y Sevillano, M. L. (Eds.) (2015). Dispositivos digitales móviles en educación. El aprendizaje ubicuo. Madrid: Narcea.

Villalustre, L., y Del Moral, M. E. (Coords.) (2016). Experiencias interactivas con realidad aumentada en las aulas. Barcelona: Octaedro.

Wei, X., Weng, D. Liu, Y., y Wang, Y. (2015). Teaching based on augmented reality for a technical creative design course. Computers \& Education, 81, 221-234. doi: 10.1016/j.compedu.2014.10.017.

Wojciechowski, R., y Cellary, W. (2013). Evaluation of learners' attitude toward learning in ARIES augmented reality environments. Computers \& Education, 68, 570-585. doi: 10.1016/j. compedu.2013.02.014. 


\section{PERFIL ACADÉMICO Y PROFESIONAL DE LOS AUTORES}

Julio Cabero Almenara. Catedrático de Tecnología Educativa de la Universidad de Sevilla, es director del Secretariado de Recursos Audiovisuales y Nuevas Tecnologías de la Universidad de Sevilla. Edito de la revista internacional Pixel Bit, Revista de Medios y Educación. Sus líneas de investigación giran en torno a las tecnologías emergentes.

E-mail: cabero@us.es

\section{DIRECCIÓN DEL AUTOR}

Facultad de Ciencias de la Educación

Calle Pirotecnia s/n

41018-Sevilla (España)

Verónica Marín Díaz. Profesora Titular de Universidad de la Facultad de Ciencias de la Educación de la Universidad de Córdoba, ha sido Directora del Máster en Educación Inclusiva de la citada universidad en el período 2012-2015. Miembro del grupo de investigación e2i de la Universidad de Córdoba y del Grupo de Tecnología Educativa de la Universidad de Sevilla. Editora de la revista internacional EDMETIC, Revista de Educación Mediática y TIC. Sus líneas de investigación giran en torno a las tecnologías emergentes.

E-mail: vmarin@uco.es

\section{DIRECCIÓN DE LA AUTORA}

Facultad de Ciencias de la Educación

Ada. San Alberto Magno s/n

14004-Córdoba (España)

Fecha de recepción del artículo: 27/03/2017

Fecha de aceptación del artículo: 28/05/2017

\section{Como citar este artículo:}

Cabero Almenara, J., y Marín-Díaz, V. (2018). Blended learning y realidad aumentada: experiencias de diseño docente. RIED. Revista Iberoamericana de Educación a Distancia, 21(1), pp. 57-74. doi: http://dx.doi.org/10.5944 ried.21.1.18719 